\title{
Case Report: Second Report of Joubert Syndrome Caused by Biallelic Variants in IFT74
}

\author{
Ke Zhongling ${ }^{1}$, Li Guoming ${ }^{1}$, Chen Yanhui ${ }^{1 *}$ and Chen Xiaoru ${ }^{2}$ \\ ${ }^{1}$ Fujian Medical University Union Hospital, Fuzhou, China, ${ }^{2}$ Guankou Hospital of Xiamen Jimei District, Xiamen, China
}

OPEN ACCESS

Edited by:

Lorenzo More

University of Central Lancashire,

United Kingdom

Reviewed by:

Dorothy Lerit,

Emory University, United States

Qingtuan Meng,

University of South China, China

*Correspondence:

Chen Yanhui

yanhui_0655@126.com

Specialty section:

This article was submitted to

Neurogenomics,

a section of the journal

Frontiers in Genetics

Received: 08 July 2021

Accepted: 09 August 2021

Published: 01 September 2021

Citation:

Zhongling K, Guoming L, Yanhui C and Xiaoru C (2021) Case Report: Second Report of Joubert Syndrome

Caused by Biallelic Variants in IFT74.

Front. Genet. 12:738157.

doi: 10.3389/fgene.2021.738157
Joubert syndrome (JBTS) is a rare ciliopathy characterized by developmental delay, hypotonia, and distinctive cerebellar and brain stem malformation called the molar tooth sign (MTS). We reported a 15-month-old female with dysmorphic features (flat nasal bridge, almond-shaped eye, and a minor midline notch in the upper lips), hypotonia, polydactyly, development delay, and MTS. Whole exome sequencing revealed biallelic heterozygous mutations c.535C > G(p.Q179E/c.853G>T) (p.E285*) in IFT74, which were inherited from the parents. So far, only one article reported JBTS associated with IFT74 gene mutation, and this is the second report of the fifth patient with JBTS due to variants in IFT74. All five patients had developmental delay, postaxial polydactyly, subtle cleft of the upper lip, hypotonia, and MTS, but notably without renal and retinal anomalies or significant obesity, and they shared the same mutation c.535C > G(p.Q179E) in IFT74, and $c .853 G>T\left(p . E 285^{*}\right)$ that we found was a new mutation in IFT74 that related with Joubert syndrome. Those findings highlight the need for the inclusion of IFT74 in gene panels for JBST testing.

Keywords: Joubert syndrome, ciliopathy, IFT74, developmental delay, polydactyly, cleft lip

\section{INTRODUCTION}

Joubert syndrome (JBTS, OMIM: P213300) is a rare, autosomal recessive ciliopathy characterized by three primary findings: a distinctive cerebellar and brain stem malformation called the molar tooth sign (MTS), hypotonia, and developmental delay (Parisi et al., 2017). About 35 ciliopathyrelated genes are known to cause JBTS (Radha Rama Devi et al., 2020); those genes encode proteins localized to the pericilia, whose dysfunction would alter cilia composition or signaling (Parisi, 2019). The Intraflagellar transport(IFT) complex is the main module for regulating cilia composition, which consists of IFT-A and IFT-B, and IFT74 is required for the stabilization of IFTB (Brown Jason et al., 2015). However, the relationship between JBST and IFT is rarely studied; thus far, only three studies have researched the correlation between JBST and IFT (Halbritter et al., 2013; Bachmann-Gagescu et al., 2015; Luo et al., 2021). In February this year, Luo et al. (2021) reported for the first time that JBST could be caused by IFT74 mutation. This paper was the second report of the fifth case of IFT74-associated JBTS; we found a new mutation in IFT74-associated JBTS and present new craniofacial dysmorphisms, which helped to expand the clinical phenotype and genotype of this syndrome.

\section{CASE REPORT}

The proband was a 15-month-old female who was referred to our department because of developmental delay. She was the first child of non-consanguineous Chinese parents. 
A

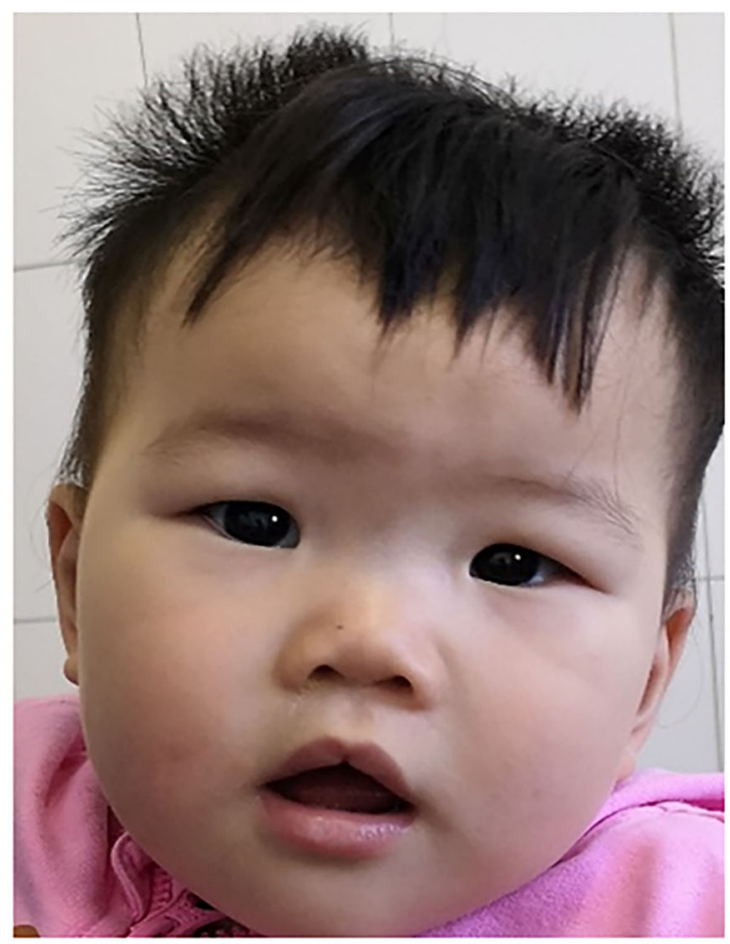

B

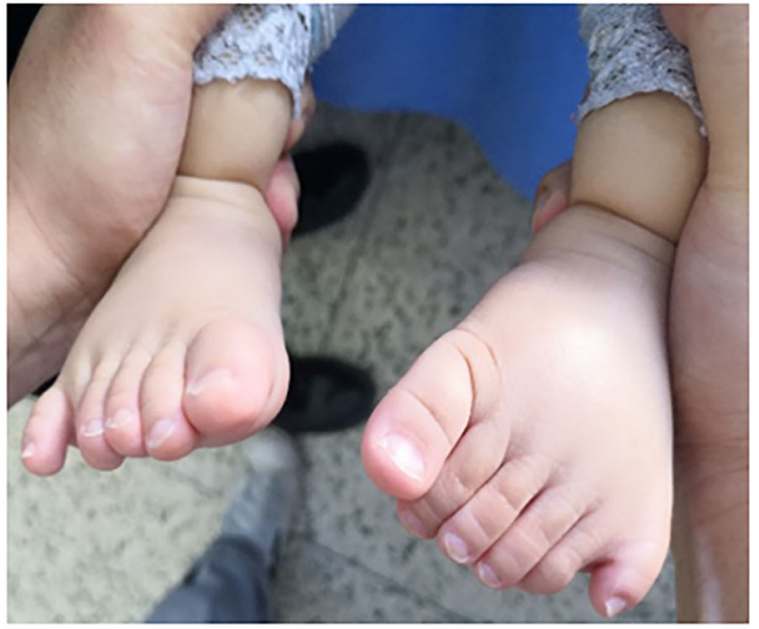

\section{C}

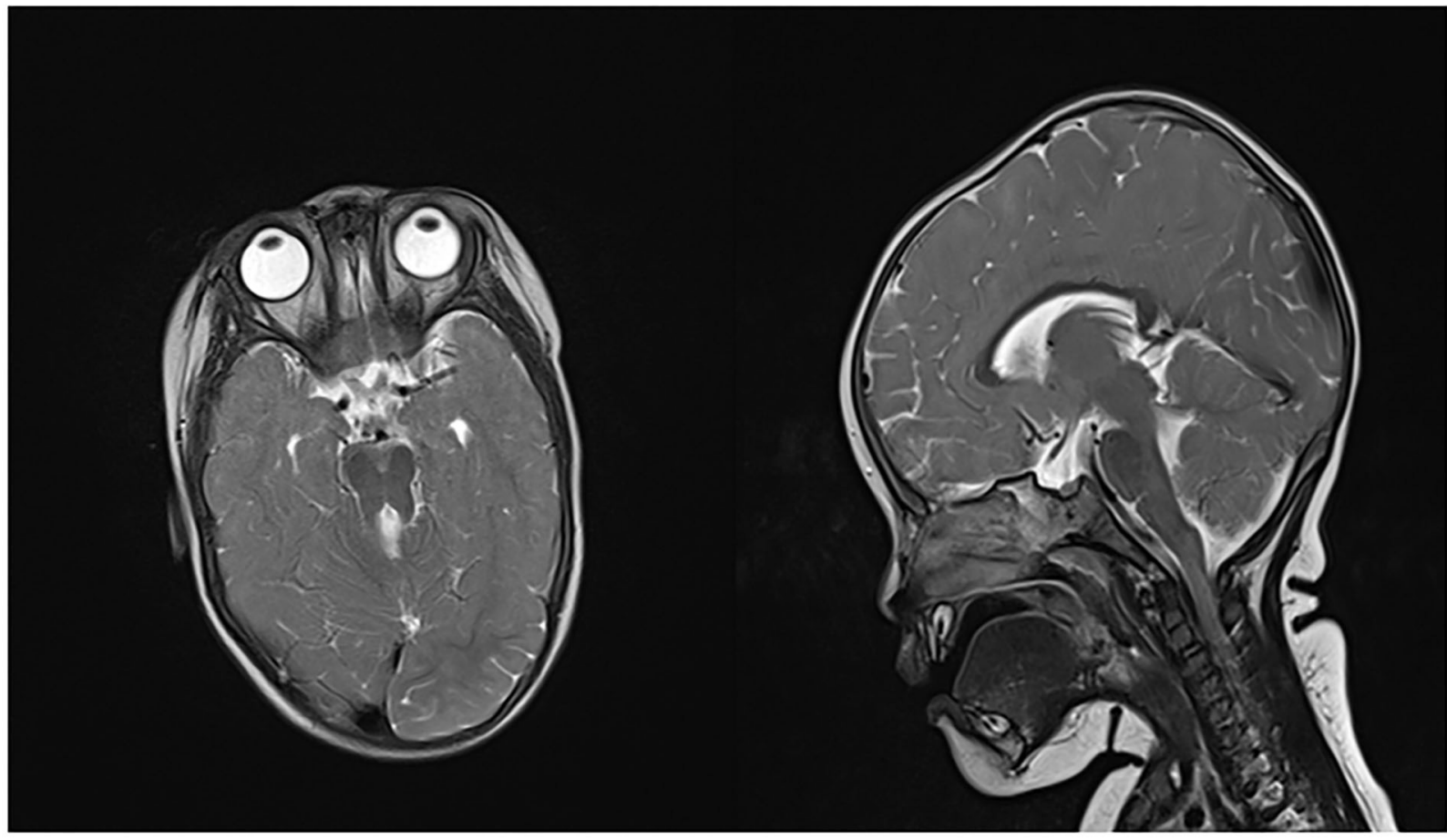

FIGURE 1 | (A) craniofacial dysmorphism (B). polydactyly (C) MTS in brain MRI. 


\section{IFT74: NM_025103.2: exon8: c.535C >G(p.Q179E)}

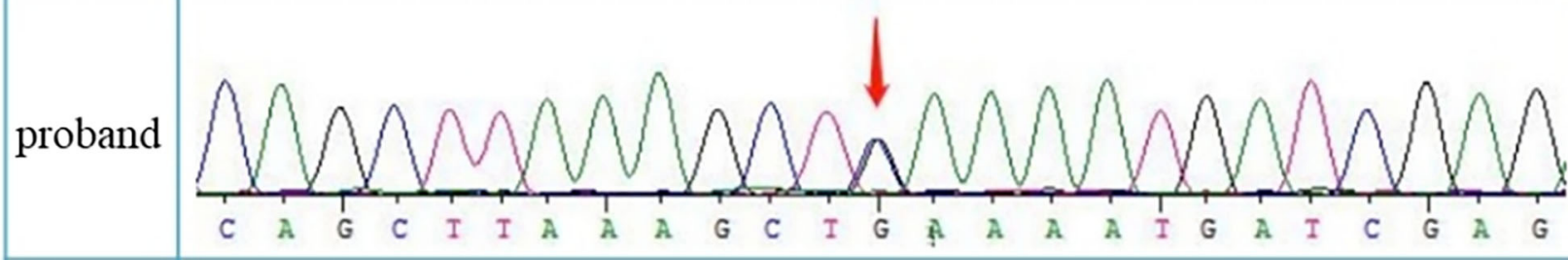

father

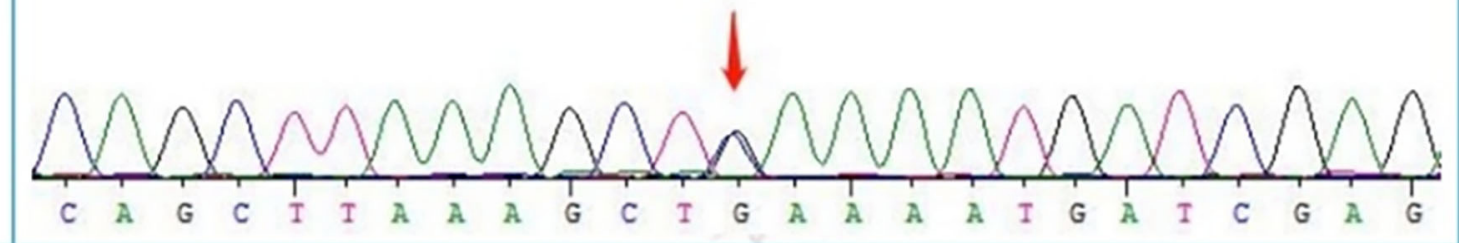

mother

\section{IFT74: NM_025103.2: exon11: c.853G>T(p.E285*)}

proband
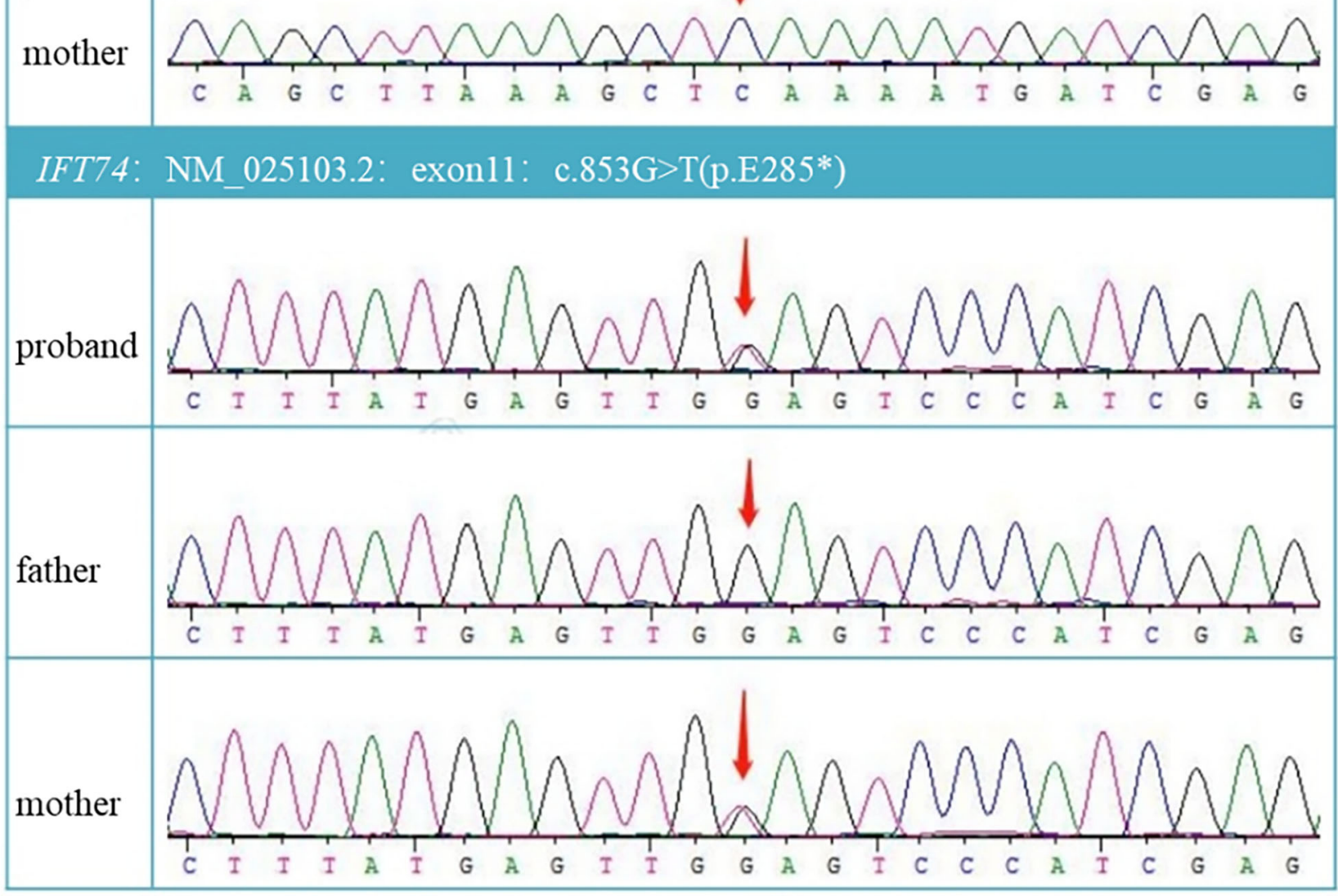

FIGURE 2 | DNA electrophoregram with the c.535C > G in exon8 and c.853G $>$ T in exon11. 
She was delivered at term via spontaneous vaginal delivery to a 35-year-old mother after an uncomplicated pregnancy. Birth weight was $3,400 \mathrm{~g}$. Immediately after birth, craniofacial dysmorphisms with a flat nasal bridge, almond-shaped eye, and a minor midline notch in the upper lips and postaxial polydactyly of the hands and feet were noticed (Figures 1A,B). She achieved rising head and sitting at 3 and 8 months old, respectively. Delayed motor development was noticed when she couldn't crawl at 1 year, and slight hypotonia of lower limbs was found. She began speaking at 1 year with slowly progressive. At the age of 9 months, she underwent a hand polydactyly excision.

There were no other family members with the presence of birth defects, developmental delay, and/or any other neurological disorders.

On examination at 15 months of age, she can stand with the assistant and speak several words. Her weight was $13 \mathrm{~kg}$, height was $82 \mathrm{~cm}$, body mass index (BMI) was $19.3 \mathrm{~kg} / \mathrm{m}^{2}$. Head circumference was $46 \mathrm{~cm}$. Investigations showed that blood count, urine routine test, biochemical test, thyroid hormones, 25 hydroxyvitamin D3 -were normal. No significant abnormalities were seen in cardiac and abdominal ultrasound examinations. An audiology evaluation was normal. Fundoscopic examination showed no retinal dystrophy, and cranial MRI showed MTS (Figure 1C). Neuropsychological development assessment was performed at the age of 16 months; the development quotient (DQ) assessment was as follows: total (64)- gross motor (52) fine motor (64) —adaptability (64) — language ability (55) — social ability (67).
With parental consent, blood was collected from the child and parents, and whole-exome sequencing was performed. Genomic DNA was extracted from the blood sample with the Blood Genomic DNA Mini Kit following manufacturer's guidelines (CWBIO). The whole-exome library was prepared using the SureSelect Human All Exon V6 (Agilent) and KAPA Hyper Prep Kit (KAPA) following the manufacturer's protocol. All sequencing was performed on the Nova seq 6,000 platform (Illumina) (Testing Service Company: The medical laboratory of Nantong Zhongke, China). Alignment and variant calling were performed with an inhouse bioinformatics pipeline. Variants with a minor allele frequency of $<0.05$ in population databases and expected to affect coding/splicing of the protein or were present in the Human Gene Mutation Database (HGMD) (Stenson et al., 2003) were included in the analysis. The American College of Medical Genetics and Genomics (ACMG) Standards and Guidelines for the interpretation of sequence variants were followed in this study.Two heterozygous variants in IFT74, NM_025103.4,c.535C>G(p.Q179E)/c.853G > T(p.E285*) were identified. Sanger sequencing showed the variants were inherited from the parents, confirming that the variants were indeed biallelic (Figures 2, 3). c.535C $>$ G(p.Q179E) was predicted to be "disease causing" with a score of 0.99 (MutationTaster), "benign" with a score of 0.126 (PolyPhen-2), and "tolerated" with a score of 0.14 (SIFT), and c.853G $>$ T (p.E285*) was predicted to be "disease causing" with a score of 1 (MutationTaster).

\section{c. $535 \mathrm{C}>\mathrm{G}$}

c. $853 \mathrm{G}>\mathrm{T}$
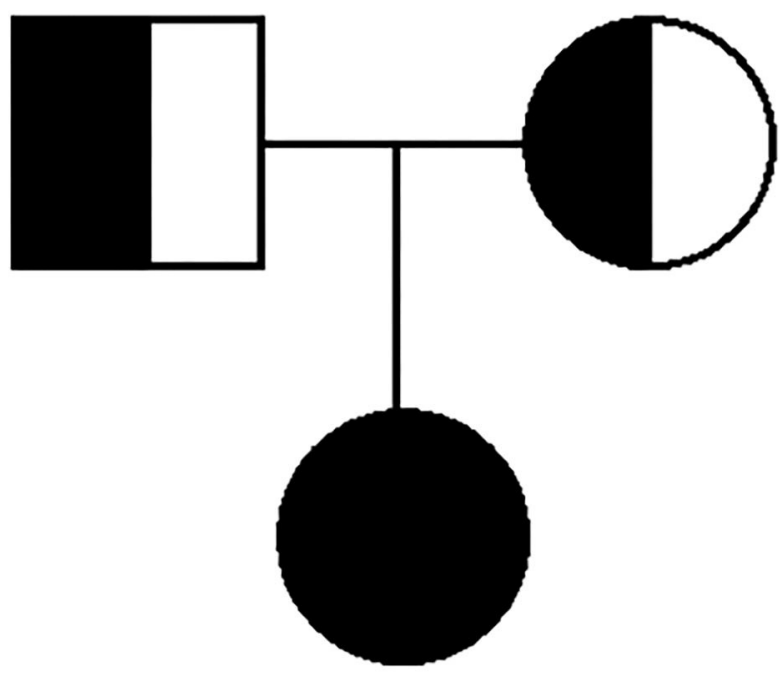

\section{c. $535 \mathrm{C}>\mathrm{G} /$ c. $853 \mathrm{G}>\mathrm{T}$}

FIGURE 3 | Pedigree of the family. 
TABLE 1 | Clinical features of patients with biallelic IFT74 variants.

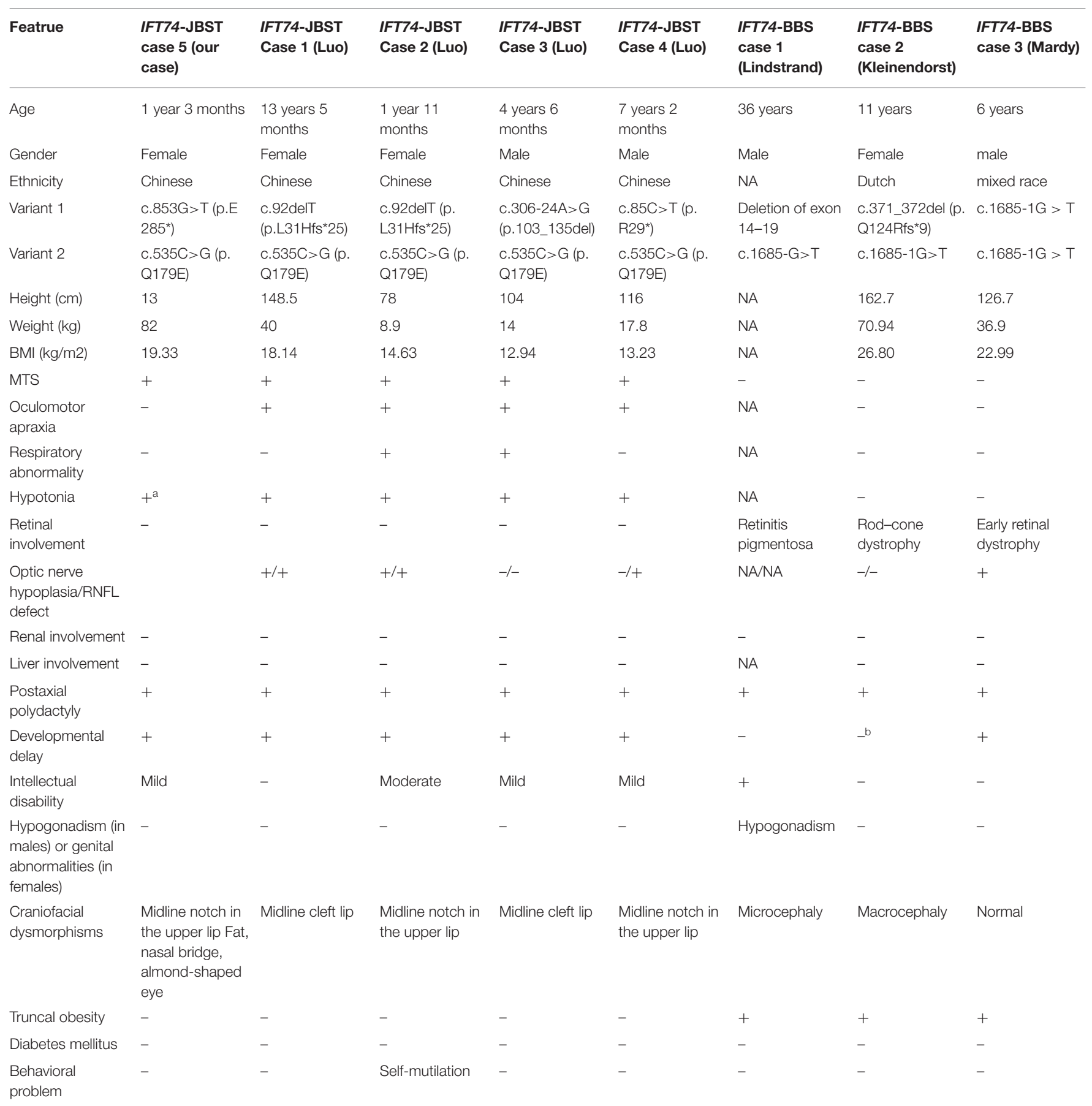

JBTS, Joubert syndrome; BBS, Bardet-Biedl syndrome; BMI, body mass index; MTS, molar tooth sign; NA, not available or not mentioned; RNFL, retinal nerve fiber layer; -, not present; + , present.

a slight hypotonia of lower limbs.

${ }^{b}$ Only speech delay in childhood. 


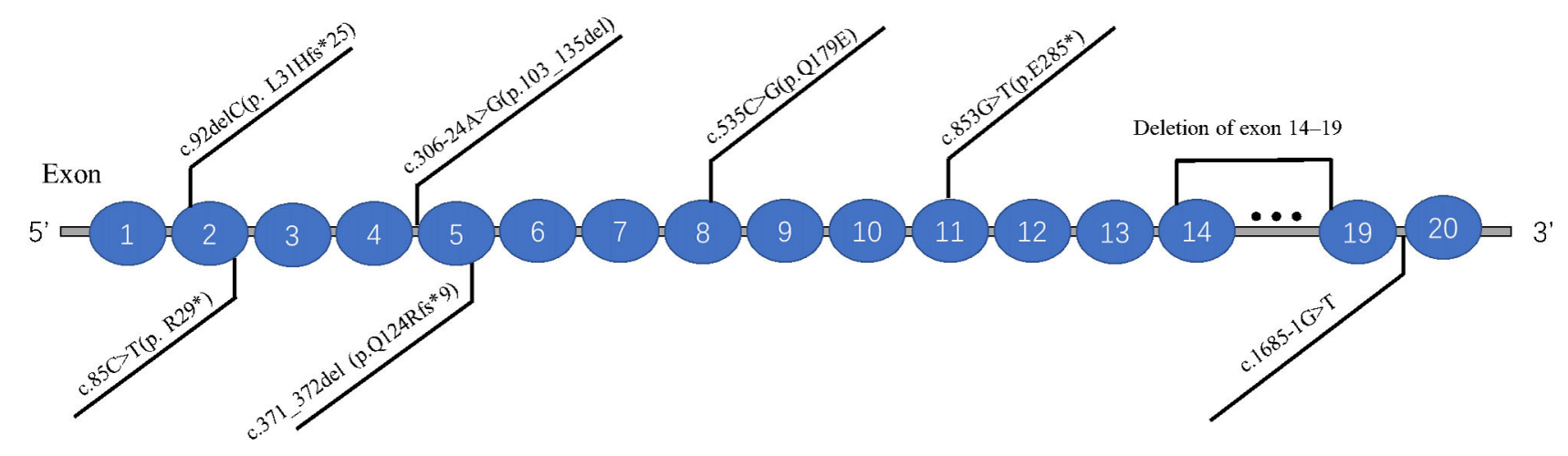

FIGURE 4 | Distribution of eight IFT74 gene mutations (transcript: NM_025103.4).

Accroding to ACMG, c.535C $>$ G(p.Q179E) was pathogenic(PS1+PS3), while c.853G $>$ T $($ p.E285*) was uncertain significant(PM2).

\section{LITERATURE REVIEW}

The databases of Pubmed, EMCC, EBSCO, and Cochrane Library were searched with the keywords of "Joubert syndrome" and "IFT74," and only one related study was found (Luo et al., 2021). And there were three articles (Lindstrand et al., 2016; Kleinendorst et al., 2020; Mardy et al., 2021) about IFT74 gene mutation related to Bardet-Biedl syndrome (BBS). The clinical features and mutations of all these cases were summary in Table 1, Figure 4.

\section{DISCUSSION}

This is the second report of IFT74 variants causing a JBTS, validating IFT74 as a JBTS gene. So far, only one previous report of four cases with JBTS caused by biallelic IFT74 variants has been published by Luo et al. (2021) this year. Luo et al. described four affected individuals from three nonconsanguineous families presenting with MTS on brain MRI, delay in global developmental milestones, postaxial polydactyly, and subtle cleft of the upper lip, all the affected individuals shared one missense variant (p.Q179E), and the pathogenic effects of the variant were evaluated by animal model, the IFT74 was identified as a JBTS-associated gene. Our patient's phenotype was consistent with Luo et al.'s report and also shared the missense variant (p.Q179E); however, the other mutation, c.853G $>$ T(p.E285*), found in our patient had not been reported in Joubert syndrome. The patient also had craniofacial dysmorphisms with a flat nasal bridge and almond-shaped eye, and these features have not been previously described in patients with a mutation of IFT74.

IFT74 gene is located on chr9: 26956412-27066134, with 20 exons (ensembl, 2021). IFT74 encodes a core intraflagellar transporter protein (IFT) that belongs to a multiprotein complex involved in the transport of ciliary proteins along axonemal microtubules. This protein binds to intraflagellar transporter protein 81 and transports microtubule proteins within the cilium, which is required for ciliogenesis (Ncbi.nlm.nih.gov., 2021). Although JBTS is known to be a recessive and heterogeneous ciliopathy (Bachmann-Gagescu et al., 2020), very little attention was paid to the relationship between IFT74 and JBTS. Until this year, Luo et al. (2021) comprehensively analyzed the cohort of 4 patients, all of which carried pathogenic mutations in the IFT74 and showed similar phenotypes, and identified IFT74 as a JBTS-associated gene.

Previous studies have suggested that IFT74 gene mutation is mainly associated with BBS, which is characterized by obesity and related complications, retinal cone-rod dystrophy, postaxial polydactyly, cognitive impairment, and renal malformations and/or renal parenchymal disease (Forsythe et al., 2018). Combined with previous reports and our findings, we found that the patients with biallelic variants in IFT74 all suffered from postaxial polydactyly and none of them had renal involvement, and the cleft lip was a special craniofacial dysmorphism in IFT74 related JBTS while retinal dystrophy and truncal obesity could be seen in all IFT74 related BBS. Intellectual disability was common in IFT74 related JBTS but with a mild or moderate degree. The mutations of IFT74 with JBTS include 2 nonsense mutations, 2 frameshift mutations, 1 splice mutations, 1 missense mutation, and were distributed in exons $2,5,8,11$, and intron 4. IFT74 mutations with BBS include 1 complete gene deletions and 1 splice mutations, distributed in exons 1419 and intron 19. Those showed that the IFT74 mutation before exon 11 were associated with JBST, while mutations after it was associated with BBS. Although the mutation was in the same gene, mutations in different locus regions may result in various protein changes, leading to these two distinct syndromes. The most common mutation type is c.535C $>$ G (p. Q179E), which was present in all the IFT74 related JBST; mechanistic studies suggested that pathogenic variants in IFT74 lead to defects in cilia length, ciliogenesis, cilia composition, and Hh signaling (Luo et al., 2021), whether c.535C > G (p. Q179E) is hot spot mutations or not need to be confirmed by future data. The mutation c.853G $>$ T (p.E285*) found in this patient had not been reported in Joubert syndrome, and there was no research on the pathogenesis of this mutation, but according to our clinical 
finding, it play a vital part in the JBTS, suggesting the loss of function due to this mutation may be pathogenic, further studies on the alter function of this mutation are needed.

In conclusion, our study reported a compound heterozygous mutation in the IFT74 gene [c.535C $>$ G(p.Q179E)/c.853G $>$ T(p.E285*)] in a Chinese family with JBTS.As far as we know, this is the second report of the fifth case in IFT74 mutation-related JBST. This patient helps to expand and clarify the clinical spectrum of IFT74-related JBST, and the cases with IFT74 mutation are summarized in Table 1. The IFT74 mutation-related JBST manifested developmental delay, postaxial polydactyly, subtle cleft of the upper lip, hypotonia, and MTS on brain MRI, but notably without renal and retinal anomalies or significant obesity. Our case also presents new craniofacial dysmorphisms of the flat nasal bridge and almond-shaped eye and the new mutation c.853G $>$ T(p.E285*) in JBST.All our findings highlight the need for the inclusion of IFT74 in gene panels for JBST testing.

\section{DATA AVAILABILITY STATEMENT}

The raw data supporting the conclusions of this article will be made available by the authors, without undue reservation.

\section{ETHICS STATEMENT}

The studies involving human participants were reviewed and approved by Fujian medical university union hospital.

\section{REFERENCES}

Bachmann-Gagescu, R., Dempsey, J. C., Bulgheroni, S., Chen, M. L., D’Arrigo, S., Glass, I. A., et al. (2020). Healthcare recommendations for Joubert syndrome. Am. J. Med. Genet. A 182, 229-249. doi: 10.1002/ajmg.a. 61399

Bachmann-Gagescu, R., Dempsey, J. C., Phelps, I. G., O’Roak, B. J., Knutzen, D. M., Rue, T. C., et al. (2015). Joubert syndrome: a model for untangling recessive disorders with extreme genetic heterogeneity. J. Med. Genet. 52, 514-522. doi: 10.1136/jmedgenet-2015-1 03087

Brown Jason, M., Cochran Deborah, A., Craige, B., Kubo, T., and Witman George, B. (2015). Assembly of IFT trains at the ciliary base depends on IFT74. Curr. Biol. 25, 1583-1593. doi: 10.1016/j.cub.2015. 04.060

ensembl (2021). Transcript: IFT74-201 ENST00000380062.10. ensembl. Available online at: https://asia.ensembl.org/Homo_sapiens/Transcript/Summary? $\mathrm{db}=$ core;g=ENSG00000096872;r=9:26947039-27066134;t=ENST00000380062 (accessed June 14, 2021).

Forsythe, E., Kenny, J., Bacchelli, C., and Beales, P. L. (2018). Managing bardet-biedl syndrome-now and in the future. Front. Pediatr. 6:23. doi: 10.3389/fped.2018. 00023

Halbritter, J., Bizet, A. A., Schmidts, M., Porath, J. D., Braun, D. A., Gee, H. Y., et al. (2013). Defects in the IFT-B component IFT172 cause Jeune and Mainzer-Saldino syndromes in humans. Am. J. Hum. Genet. 93, 915-925. doi: 10.1016/j.ajhg.2013. 09.012
Written informed consent to participate in this study was provided by the participants' legal guardian/next of kin. Written informed consent was obtained from the individual(s), and minor(s)' legal guardian/next of kin, for the publication of any potentially identifiable images or data included in this article.

\section{AUTHOR CONTRIBUTIONS}

KZ contributed to the acquisition, analysis of data, and writing the first draft. CY contributed to the conception of the work and revised the paper. LG contributed to collect the data and communicated with the pateints family. CX contributed to search information of this disease.

\section{FUNDING}

This work was supported by the National Key Research and Development Program of China (NO.2016YFC1306204) and the Joint Funds for the innovation of science and Technology, Fujian province (NO. 2017Y9043).

\section{ACKNOWLEDGMENTS}

The authors would like to thank the patient and parents for their cooperation and consent to this study.

Kleinendorst, L., Alsters, S. I. M., Abawi, O., Waisfisz, Q., Boon, E. M. J., van den Akker, E. L. T., et al. (2020). Second case of Bardet-Biedl syndrome caused by biallelic variants in IFT74. Eur. J. Hum. Genet. 28, 943-946. doi: 10.1038/s41431-0200594-z

Lindstrand, A., Frangakis, S., Carvalho, C. M., Richardson, E. B., McFadden, K. A., Willer, J. R., et al. (2016). Copy-number variation contributes to the mutational load of bardet-biedl syndrome. Am. J. Hum. Genet. 99, 318-336. doi: 10.1016/j.ajhg.2015. 04.023

Luo, M., Lin, Z., Zhu, T., Jin, M., Meng, D., He, R., et al. (2021). Disrupted intraflagellar transport due to IFT74 variants causes Joubert syndrome. Genet. Med. 23, 1041-1049. doi: 10.1038/s41436-021-0 1191-0

Mardy, A. H., Hodoglugil, U., Yip, T., and Slavotinek, A. M. (2021). Third case of Bardet-Biedl syndrome caused by a biallelic variant predicted to affect splicing of IFT74. Clin Genet. 100, 93-99. doi: 10.1111/cge.13962

Ncbi.nlm.nih.gov. (2021). IFT74 Intraflagellar Transport 74 [ Homo sapiens (human) ]. Gene. Available online at: https://www.ncbi.nlm.nih.gov/gene/ 80173 (accessed June 14, 2021).

Parisi, M., Glass, I., Adam, M. P., Ardinger, H. H., Pagon, R. A., Wallace, S. E., et al. (eds). (2017). Joubert Syndrome. Seattle, WA: University of Washington. Available online at: https://pubmed.ncbi.nlm.nih.gov/20301500/ (accessed June 14, 2021)

Parisi, M. A. (2019). The molecular genetics of Joubert syndrome and related ciliopathies: the challenges of genetic and phenotypic heterogeneity. Transl. Sci. Rare Dis. 4, 25-49. doi: 10.3233/TRD-1 90041 
Radha Rama Devi, A., Naushad, S. M., and Lingappa, L. (2020). Clinical and molecular diagnosis of joubert syndrome and related disorders. Pediatr. Neurol. 106, 43-49. doi: 10.1016/j.pediatrneurol.2020. 01.012

Stenson, P. D., Ball, E. V., Mort, M., Phillips, A. D., Shiel, J. A., Thomas, N. S. T., et al. (2003). Human Gene Mutation Database (HGMD $®)$ : 2003 update. Human. Mutation 21, 577-581. doi: 10.1002/humu. 10212

Conflict of Interest: The authors declare that the research was conducted in the absence of any commercial or financial relationships that could be construed as a potential conflict of interest.
Publisher's Note: All claims expressed in this article are solely those of the authors and do not necessarily represent those of their affiliated organizations, or those of the publisher, the editors and the reviewers. Any product that may be evaluated in this article, or claim that may be made by its manufacturer, is not guaranteed or endorsed by the publisher.

Copyright (C) 2021 Zhongling, Guoming, Yanhui and Xiaoru. This is an open-access article distributed under the terms of the Creative Commons Attribution License (CC $B Y)$. The use, distribution or reproduction in other forums is permitted, provided the original author(s) and the copyright owner(s) are credited and that the original publication in this journal is cited, in accordance with accepted academic practice. No use, distribution or reproduction is permitted which does not comply with these terms. 